\title{
The Gain Stabilization of SiPM
}

\section{Ivo Polák ${ }^{1}$ on behalf of the CALICE Collaboration}

Institute of Physics of the Academy of Sciences of the Czech Republic

Na Slovance 2, CZ - 18221 Prague 8, Czech Republic

E-mail: polakiefzu.cz

\begin{abstract}
We have designed and developed the adaptive power supply to keep the gain stability of SiPMs in the Analog Hadron Calorimeter of the CALICE ILC detector stable at the level of $1 \%$. This power supply compensates automatically the bias voltage for temperature variations in the range of $5-40{ }^{\circ} \mathrm{C}$. It operates in the bias voltage range $15-90 \mathrm{~V}$ suitable for most of SiPMs on the market. We demonstrate the performance of the gain stability with a prototype that was produced in industry after measurements with a test board. We checked the gain stability for several types of SiPMs from three producers - Hamamatsu, KETEK and CPTA.
\end{abstract}

Technology and Instrumentation in Particle Physics 2014

2-6 June, 2014

Amsterdam, the Netherlands

${ }^{1}$ Speaker 


\section{Introduction}

The gain $G$ of SiPMs (MPPCs) depends both on the bias voltage $V_{\text {bias }}$ and on the temperature $T$. For thestable operation of calorimeteres the gain needs to be kept constant. In the ILC calorimeter with millions of channels this is a challenging task. It is, therefore, desirable to compensate the gain automatically for temperature variations by readjusting the bias voltage.

As part of the AIDA framework, the University of Bergen and the Institute of Physics in Prague have designed and developed a suitable adaptive power supply. Taking into account uncertainties on the temperature measurement and the power supply accuracy, the goal consists of keeping $G$ constant within $1 \%$ over a temperature range of $\Delta T \pm 5{ }^{\circ} \mathrm{C}$ at the nominal temperature of $25{ }^{\circ} \mathrm{C}$. We later showed that the circuit keeps the $1 \%$ gain stability in the larger range $5-40{ }^{\circ} \mathrm{C}$.

First, we need to measure SiPM gain versus $T$ and gain versus $V_{\text {bias. }}$. Then we determine $\mathrm{d} V_{\text {bias }} / \mathrm{d} T$ from the condition for the constant gain. We apply the compensation in the $V_{\text {bias }}$ regulator and demonstrate the proof of principle.

\section{Design considerations}

The bias voltage regulator test board should satisfy the following requirements:

- Provide stable regulated output DC voltage from $15 \mathrm{~V}$ to $90 \mathrm{~V}$

- Achieve the stability better than $5 \mathrm{mV}$ and keep the temperature influence to less than $1 \mathrm{mV} /{ }^{\circ} \mathrm{C}\left(100 \mathrm{ppm} /{ }^{\circ} \mathrm{C}\right)$ in the entire range of $V_{\text {bias }}$

- Trim the signal with a slope $\mathrm{d} V_{\text {bias }} / \mathrm{d} T$ of 10 to $100 \mathrm{mV} /{ }^{\circ} \mathrm{C}$

- Have an ON/OFF ability and provide a soft start/stop

- Provide analog settings with later possibility to implement digital settings with DAC.

\section{SiPM test setup}

To measure the temperature dependence of the gain we used a climate chamber at CERN that is stable within $0.2^{\circ} \mathrm{C}$. A SiPM was placed to the socket on a preamplifier PCB (see Fig.1).
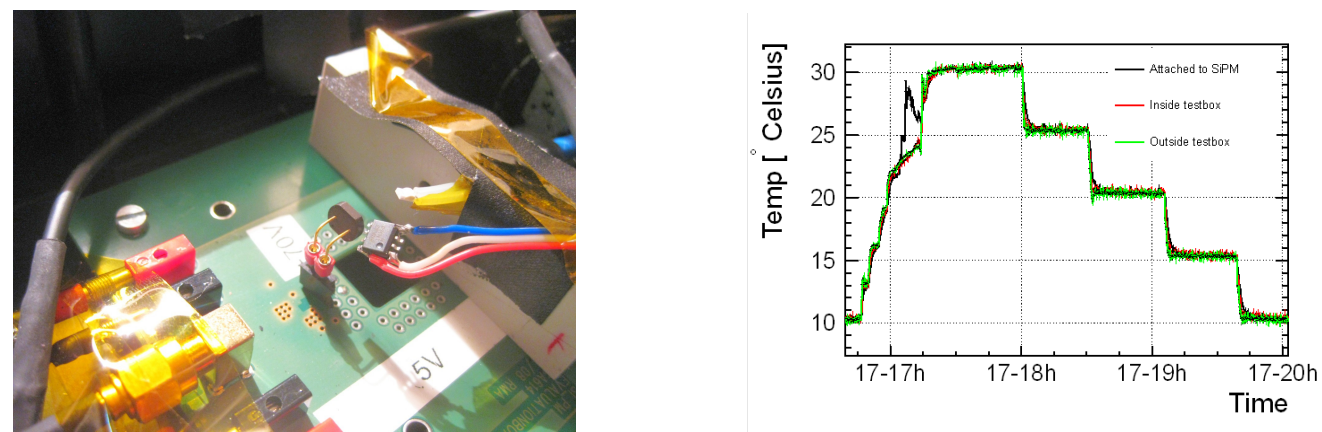

Fig.1. SiPM in socket placed on preamplifier PCB with temperature sensor LM35 (left). At right there is a temperature profile of one measurement in the climate chamber. 
We worked with SiPMs from three manufacturers: CPTA, Hamamatsu and KETEK (the list of SiPMs and MPPCs is given in Tab. 1). We utilize the default T3B calibration procedure [1] to extract the single photo-electron spectra of SiPMs. DAQ includes preamplifier and 1.25 Gsps digital oscilloscope (picoscope) [2], which was set to acquire 50,000 events per run. The algorithm identifies the signal of a single photo-electron and it is subsequently capable of decomposing any SiPM signal into the cumulative signal of single photo-electrons. SiPMs were illuminated by blue LED driven from a pulse generator.

We used three thermistors PT1000 to monitor ambient temperature around SiPM. Another temperature sensor LM35D (Texas instruments) placed close to a SiPM under the test provides a signal $10 \mathrm{mV} /{ }^{\circ} \mathrm{C}$ to the regulator for the correction of the output bias voltage.

\begin{tabular}{|c|c|c|c|c|c|c|}
\hline $\begin{array}{l}\text { Manufacturer } \\
\text { and Type \# }\end{array}$ & $\begin{array}{c}\text { Sensitive area } \\
{\left[\mathrm{mm}^{2}\right]}\end{array}$ & $\begin{array}{c}\text { Pixel pitch } \\
{[\mu \mathrm{m}]}\end{array}$ & \#pixels & $\begin{array}{c}\text { Typical } \\
V_{\text {bias }}[\mathrm{V}]\end{array}$ & $\begin{array}{l}\text { Typical } \\
G\left[\times 10^{5}\right]\end{array}$ & Serial \# \\
\hline \multicolumn{7}{|l|}{ Hamamatsu } \\
\hline S10943-8584(X) & $1 \times 1$ & 50 & 400 & 71.69 & 7.49 & 11759 \\
\hline S10943-8584(X) & $1 \times 1$ & 50 & 400 & 71.57 & 7.49 & 11766 \\
\hline S10943-8584(X) & $1 \times 1$ & 50 & 400 & 71.50 & 7.48 & 11770 \\
\hline S10943-8584(X) & $1 \times 1$ & 50 & 400 & 71.33 & 7.48 & 11771 \\
\hline Sample A & $1 \times 1$ & 20 & 2500 & 66.7 & 2.3 & A1 \\
\hline Sample B & $1 \times 1$ & 20 & 2500 & 73.3 & 2.3 & B1 \\
\hline Sample A & $1 \times 1$ & 15 & 4440 & 67.2 & 2.0 & A2 \\
\hline Sample B & $1 \times 1$ & 15 & 4440 & 74.0 & 2.0 & B2 \\
\hline \multicolumn{7}{|l|}{ CPTA } \\
\hline & $1 \times 1$ & 40 & 796 & 33.4 & 7.1 & 857 \\
\hline & $1 \times 1$ & 40 & 796 & 33.1 & 6.3 & 922 \\
\hline & $1 \times 1$ & 40 & 796 & 33.3 & 6.3 & 975 \\
\hline & $1 \times 1$ & 40 & 796 & 33.1 & 7.0 & 1065 \\
\hline & $1 \times 1$ & 40 & 796 & 33.3 & 14.6 & 1677 \\
\hline \multicolumn{7}{|l|}{ KETEK } \\
\hline MP15 V6 ? & $2(1.2 \times 1.2)$ & $15 ?$ & ? & -28 & $?$ & W8 \\
\hline MP20 V4 ? & $3 \times 3$ & $20 ?$ & $?$ & -28 & $?$ & W12 \\
\hline
\end{tabular}

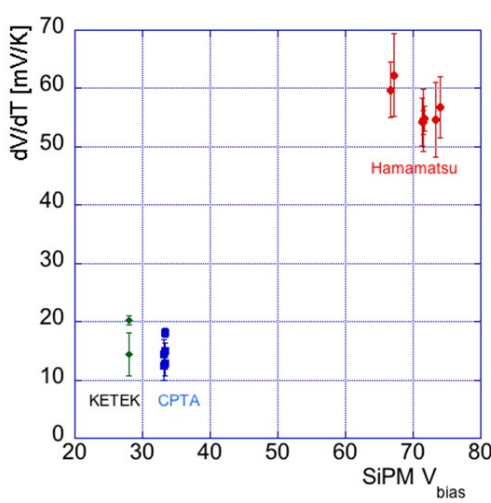

Tab. 1. A list of SiPM (MPPC) we tested (left).

Fig. 2. The compensation slope $\mathrm{d} V_{\text {bias }} / \mathrm{d} T$ versus bias $V_{\text {bias }}$ for three types of SiPMs (right).

All measurements were performed in the temperature range of 10 to $30^{\circ} \mathrm{C}$. The results of the gain dependence on temperature and bias voltage were recalculated to the $\mathrm{d} V_{\text {bias }} / \mathrm{d} T$ compensation slope for each SiPM and were used in the design of the adaptive bias voltage regulator test board. After the circuit construction, we measured with it a subset of four SiPMs/MPPCs again in the extended temperature range $5-40^{\circ} \mathrm{C}$. These were Hamamatsu \#11759, CPTA \#857 and \#1677 and KETEK \#W12.

\section{Adaptive power regulator}

The power part of the analogue HV regulator uses two HV MOSFETs in a totem pole configuration. In the control part, the output voltage is divided by ten using precision resistors and attached to the feedback input of the error amplifier. The reference input of the amplifier is connected to a multi-turn potentiometer which is connected to a precision voltage reference LT1021-10. The voltage reference is trimmed to $10.000 \mathrm{~V}$ and corresponds to $100 \mathrm{~V}$ at the output of the regulator. Multi-turn potentiometer sets output voltage in range $15-100 \mathrm{~V}$. The error amplifier drives the gate of the lower MOSFET. Loop stability is achieved by means of a third order compensation network. Temperature sensor LM35D (Texas instruments) placed close to SiPM under the test provides a signal $10 \mathrm{mV} /{ }^{\circ} \mathrm{C}$ for the correction of the output voltage. We used analogue design which omits problems with nonlinearity of ADC/DAC conversion. In 
any case for this application an equivalent of effective 16 bits resolution is needed. The design can be extended to the control from a PC with a DAC module to set the nominal output voltage.
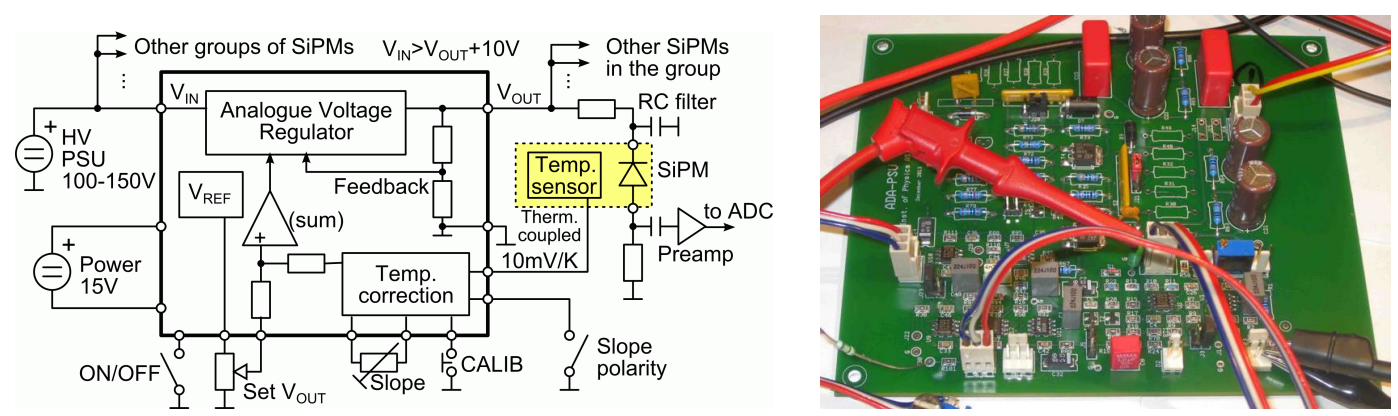

Fig.3. Principal scheme of ADApower regulator (left) and this circuit on the PCB (right).

\section{Results of the gain stabilization}
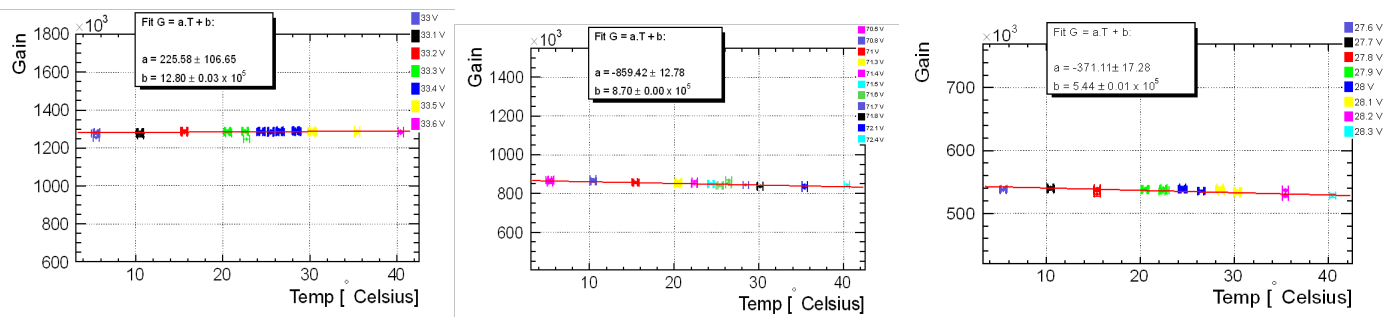

Fig.4. Gain after the compensation for: CPTA1677, Hamamatsu11759 and Ketek W12.

The gain stability of SiPMs with compensation is well below $1 \%$, ranging from $0.05 \%$ for Ketek W12 to $0.9 \%$ for Hamamastu 11759. Adjustment range for bias voltage is $0.7 \mathrm{~V}$ for CPTA \& KETEK and 1.9 V for Hamamatsu SiPMs.

\section{Conclusion}

We measured the $\mathrm{d} G / \mathrm{d} V_{\text {bias }}$ and $\mathrm{d} G / \mathrm{d} T$ dependences [3] for 15 SiPMs from 3 manufacturers. From these measurements we extracted the compensation slope $\mathrm{d} V_{\text {bias }} / \mathrm{d} T$. The adaptive power regulator described in this contribution can stabilize the SiPM gain below $1 \%$ in the temperature range 5 to $40{ }^{\circ} \mathrm{C}$.

We acknowledge the support of Grant Agencies².

\section{References}

[1] C. Soldner, The Time Development of Hadronic Showers and the T3B Experiment., Ph.D thesis, Ludwig Maximilian Universität München, 2013.

[2] PicoTech homepage: http://www.picotech.com/ .

[3] G. Eigen et al., Proceedings of the LCWS 2013 http://arxiv.org/pdf/1403.8104v1.pdf .

\footnotetext{
${ }^{2}$ EC as INFRA project no. 262025 AIDA, Ministry of Education, Youth and Sports, Czech Republic, project INGO II - LG14033 and Norwegian Research Council.
} 